Präv Gesundheitsf 2022 $17: 178-184$ https://doi.org/10.1007/s11553-021-00846-9 Eingegangen: 19. Dezember 2020

Angenommen: 22. März 2021

Online publiziert: 21. April 2021

(c) Der/die Autor(en) 2021

\author{
Caroline Koinzer ${ }^{1}$ (D) Kathleen Hirsch ${ }^{2}$ Ernst Peter Richter ${ }^{3} \cdot$ Hendrik Berth $^{3}$ \\ ' Fakultät Gesundheits- und Pflegewissenschaften, Westsächsische Hochschule Zwickau, Zwickau, \\ Deutschland \\ ${ }^{2}$ Krankenpflegeschule, Unternehmensbildungszentrum, Harzklinikum D.-C.-Erxleben GmbH, \\ Quedlinburg, Deutschland \\ ${ }^{3}$ Medizinische Fakultät Carl Gustav Carus, Bereich für Psychosoziale Medizin und \\ Entwicklungsneurowissenschaften, Forschungsgruppe Angewandte Medizinische Psychologie und \\ Medizinische Soziologie, Technische Universität Dresden, Dresden, Deutschland
}

\title{
Psychische Gesundheit von Studierenden
}

\section{Ein Vergleich zwischen Gesundheits- und Pflegemanagementstudierenden der Westsächsischen Hochschule Zwickau (WHZ) und Medizinstudierenden der Technischen Universität (TU) Dresden}

Bei der Aufrechterhaltung der psychischen Gesundheit sind u.a. verschiedene Lebensabschnitte mit ihren jeweiligen Anforderungen zu berücksichtigen. Bei Betrachtung von jungen Erwachsenen wurde deutlich, dass Studierende im Vergleich zu gleichaltrigen Erwerbspersonen psychisch stärker belastet zu sein scheinen. Besonders bei Medizinstudierenden ist die Prävalenz für psychische Auffälligkeiten hoch. Aufgrund der unzureichenden Studienlage galt es herauszufinden, ob Studierende der Medizin, im Vergleich zu Studierenden in der gleichen Branche, psychisch stärker belastet sind.

\section{Hintergrund und Fragestellung}

Die psychische Gesundheit ist eine wesentliche Voraussetzung für die Bewältigung von Aufgaben auf individueller und gesellschaftlicher Ebene [11]. Anders als bei somatischen Erkrankungen sind von psychischen Störungen häufiger jüngere Menschen im produktiven Alter betroffen [18]. Insbesondere die Studienzeit gilt als Phase der persönli- chen Entwicklung, in welcher die jungen Erwachsenen mit spezifischen Anforderungen konfrontiert werden [16]. Diese zeigen sich insbesondere in Prüfungsdichte, Bewältigung der Stoffmenge und Zeitdruck [19]. Wenn die Studierenden für diese Anforderungen keine adäquaten Bewältigungs- bzw. Lösungsstrategien entwickeln, besteht die Gefahr der Entwicklung von psychischen Störungen [16]. Die Sonderauswertung des Gesundheitsreports der Techniker Krankenkasse aus dem Jahr 2015 hat deutlich gemacht, dass psychische Störungen im Gegensatz zu anderen Erkrankungen bei Studierenden häufiger sind als bei gleichaltrigen Erwerbspersonen [26]. Sie entstehen infolge von dauerhafter Fehlbeanspruchung als negative Auswirkung von Belastungen im Studium und Privatleben. Dies kann sich wiederum negativ auf den Studienerfolg auswirken [24].

Um Interventionsmaßnahmen anbieten zu können, sind differenzierte Analysen der psychischen Belastung von Studierenden und deren Ursachen nötig. Faktoren, welche die psychische Gesundheit beeinträchtigen können, sind z.B. finanzielle Schwierigkeiten, riskanter Alkoholkonsum [1, 2, 9] und das Geschlecht. Bailer et al. [1] berichten, dass Frauen ein fast doppelt so hohes Risiko $(\mathrm{OR}=1,8)$ für eines der im "patient health questionnaire“ erfragten Syndrome (z.B. Panik- oder depressives Major-Syndrom) haben als Männer, mit Ausnahme des Alkoholsyndroms, bei welchem umgekehrte Tendenzen zu beobachten sind. Auch bezüglich des Studienfachs konnten bereits Unterschiede im Hinblick auf die psychische Gesundheit empirisch gefunden werden: Medizinstudierende gelten internationalen Studien zufolge als besonders belastet.

Das psychische Befinden von $\mathrm{Me}$ dizinstudierenden wird seit mehreren Jahrzehnten v.a. im angloamerikanischen Sprachraum erforscht. Dyrbye et al. [7] legten einen systematischen Review zu psychischen Beeinträchtigungen von Medizinstudierenden vor, welcher US-amerikanische und kanadische Studierende berücksichtigte. Hope und Henderson [17] erarbeiteten einen systematischen Review zu psychischen Beeinträchtigungen von Medizinstudierenden im europäischen Raum sowie in anderen englischsprachigen Ländern außerhalb von Nordamerika. Beide Re- 


\begin{tabular}{|c|c|c|c|}
\hline & & \multicolumn{2}{|l|}{ Studienfach } \\
\hline & & $\begin{array}{l}\text { Gesundheits- und } \\
\text { Pflegemanagement }\end{array}$ & Medizin \\
\hline \multicolumn{2}{|c|}{ Absolute Häufigkeit (n) } & 103 & 155 \\
\hline \multicolumn{2}{|c|}{ Relative Häufigkeit } & $100,0 \%$ & $100,0 \%$ \\
\hline \multirow[t]{4}{*}{ Geschlecht } & \multirow{2}{*}{ Weiblich $(n)$} & 91 & 113 \\
\hline & & $88,3 \%$ & $72,9 \%$ \\
\hline & \multirow[t]{2}{*}{ Männlich (n) } & 12 & 42 \\
\hline & & $11,7 \%$ & $27,1 \%$ \\
\hline \multirow[t]{2}{*}{ Alter (Jahre) } & Arithmetisches Mittel & 21,6 & 22,1 \\
\hline & Standardabweichung & 4,3 & 3,7 \\
\hline
\end{tabular}

views kamen zu dem Ergebnis, dass Medizinstudierende im Vergleich zur gleichaltrigen Allgemeinbevölkerung höhere Prävalenzraten bezüglich Depression, Angst und Distress aufwiesen [7, 17]. Dinkel et al. [6] berichten auf Grundlage zahlreicher gesichteter Studien Prävalenzraten zwischen 20 und $50 \%$ für psychische Beeinträchtigungen bei Medizinstudierenden, wenn als Indikatoren unspezifischer Distress und Angst herangezogen werden. Auch neuere Untersuchungen belegen dies. In der Studie von Eissler et al. [8] sind Angst und depressive Störungen bei Studierenden im Vergleich zur Allgemeinbevölkerung doppelt so häufig. Lutz-Kopp et al. [20] finden bei mehr als der Hälfte der befragten Studierenden eine niedriges Wohlbefinden und eine geringe Stressresilienz.

Bei Betrachtung von Studienfächern, welche ebenfalls der Gesundheitsbranche angehören, sind gleichermaßen hohe Prävalenzraten zu finden. Bíró et al. [3] fanden heraus, dass knapp ein Fünftel $(19 \%)$ der von ihnen untersuchten Gesundheitswissenschaftsstudierenden unter psychischem Distress litt. Häufig arbeiten sowohl Medizinstudierende als auch Studierende der Gesundheits- und Pflegewissenschaften nach Studienabschluss in Settings des Gesundheitswesens. Dennoch unterscheidet sich ihr Studienfach und das Aufgabengebiet deutlich. Das Medizinstudium ist im Vergleich $\mathrm{zu}$ anderen Fächern wesentlich stärker strukturiert, eine deutlich größere Zahl von Pflichtveranstaltungen (mit Vor- und Nachbereitungsaufwand) und häufige Lernstandskontrollen mit sehr umfangreichen Stoffmengen prä- gen den zeitintensiven Studienalltag. Der Zugang zum Medizinstudium ist limitiert (Numerus clausus), daher besteht die Population der Medizinstudierenden aus Personen mit überdurchschnittlichen Abiturergebnissen. Während nach Abschluss viele der Ärztinnen und Ärzte klinisch am Patienten tätig werden, sind Absolventen der Gesundheits- und Pflegewissenschaften, des Gesundheitsmanagements usw. häufig eher administrativ tätig, woraus unterschiedliche Belastungen im Berufsalltag resultieren können. daher, Aspekte psychischer Gesundheit von Studierenden unterschiedlicher Studienfächer, die jedoch zu der gleichen Branche gehören, $\mathrm{zu}$ untersuchen und zu vergleichen.

\section{Studiendesign und Untersuchungsmethoden}

\section{Studienpopulation}

Die Studie wurde in Kooperation mit der Forschungsgruppe Angewandte Medizinische Psychologie und Soziologie des eigenständigen Bereichs Psychosoziale Medizin und Entwicklungsneurowissenschaften der Medizinischen Fakultät Carl Gustav Carus Dresden durchgeführt. Untersucht wurden Studierende der beiden Studiengänge Gesundheits- und Pflegemanagement an der Westsächsischen Hochschule Zwickau (WHZ). An diesem Standort bestand die Grundgesamtheit aus 194 Studierenden aus dem ersten, dritten und fünften Semester. Außerdem wurden Humanmedizinstudierende der Technischen Universität (TU) Dres-
Das Globalziel der Untersuchung war den aus dem dritten Semester befragt, in welchem zum Befragungszeitpunkt 227 Personen studierten.

Die Teilnahme an der Befragung war freiwillig. Indem die Studierenden den Fragebogen ausfüllten, willigten sie ein, an der Untersuchung teilzunehmen. Alle beschriebenen Untersuchungen am Menschen wurden mit Zustimmung der zuständigen Ethikkommission (Ethikkommission der Technischen Universität Dresden, EK199052014), im Einklang mit nationalem Recht sowie gemäß der Deklaration von Helsinki von 1975 (in der aktuellen, überarbeiteten Fassung) durchgeführt.

\section{Erhebungsmethode}

Für die Datenerhebung wurde die wissenschaftliche Fragebogenmethode angewendet. Es handelte sich dabei um einen vollstandardisierten elektronischen Fragebogen, welcher online von den Studierenden bearbeitet wurde. Die Erhebung fand zwischen dem 17.10.2018 und dem 28.11.2018 statt. Der Erhebungszeitraum am Beginn des Wintersemesters wurde gewählt, da an beiden Studienorten noch keine Prüfungen stattfanden. Um die psychische Gesundheit zu ermitteln, wurden die Prüfungsängstlichkeit, die persönliche Situation und die subjektiv wahrgenommene Belastung durch das Studium untersucht. Der Fragebogen beinhaltete die folgenden validierten Erhebungsinstrumente:

TAI-G (Kurzform). Die Erfassung der Prüfungsängstlichkeit erfolgte mithilfe der Kurzform des „German Test Anxiety Inventory“ (TAI-G-Kurzskala). Das TAI-G ist die übersetzte, erweiterte und modifizierte Version des „Test Anxiety Inventory“ (TAI) von Spielberger [22]. Es setzt sich in der Originalversion aus 30 Items [15] und in der Untersuchung verwendeten verkürzten Form aus 15 Items zusammen [28]. Diese werden inhaltlich in vier Dimensionen untergliedert, welche eine gute interne Konsistenz aufweisen: Aufgeregtheit (Chronbachs $\alpha=0,88$ ), Besorgtheit (Chronbachs $\alpha=0,87$ ), Mangel an Zuversicht (Chronbachs $\alpha=0,85$ ) und Interferenz (Chronbachs $a=0,71$ ). Die Antworten werden mit numerischen 
Präv Gesundheitsf 2022 · 17:178-184 https://doi.org/10.1007/s11553-021-00846-9

(c) Der/die Autor(en) 2021

\section{Koinzer $\cdot$ K. Hirsch $\cdot$ E. P. Richter $\cdot$ H. Berth}

\section{Psychische Gesundheit von Studierenden. Ein Vergleich zwischen Gesundheits- und Pflegemanagementstudierenden der Westsächsischen Hochschule Zwickau (WHZ) und Medizinstudierenden der Technischen Universität (TU) Dresden}

\section{Zusammenfassung}

Hintergrund. Die Studienzeit ist eine Phase, in der die jungen Erwachsenen mit spezifischen Anforderungen konfrontiert werden. Studierende der Medizin gelten als besonders psychisch belastet. Untersuchungen, in denen sie diesbezüglich mit Studienfächern der gleichen Branche verglichen werden, sind jedoch rar.

Fragestellung. Ziel der Untersuchung war es, die psychische Gesundheit von Medizinstudierenden sowie von Gesundheitsund Pflegemanagementstudierenden zu untersuchen und zu vergleichen.

Material und Methoden. Untersucht wurden Studierende der Studiengänge Gesundheitssowie Pflegemanagement (WHZ) und Medizinstudierende der (TU Dresden). Verschiedene Aspekte psychischer Gesundheit wurden mithilfe eines Online-Fragebogens erhoben. Dieser beinhaltete die Instrumente zur Erfassung der Prüfungsängstlichkeit (TAIG-Kurzskala) und psychischen Belastung (BSI18), sowie ein Item zur Ermittlung der subjektiv wahrgenommenen Studienbelastung. 258 Studierende nahmen an der Befragung teil.

Ergebnisse. Es konnten keine signifikanten Unterschiede hinsichtlich des Gesamtscores der Prüfungsängstlichkeit und der psychischen Belastung zwischen den Studierenden beider Studienfächer festgestellt werden. Dahingegen gaben Medizinstudierende an, dass sie im Vergleich zu Studierenden der anderen beiden Studienfächer eine signifikant höhere Studienbelastung wahrnehmen.
Schlussfolgerung. Die ermittelten Gesamtscores lagen teilweise über den Werten in vergleichbaren Studien. Die Studierenden sollten bereits präventiv unterstützt werden, um eigene Bewältigungsstrategien zu entwickeln. Nicht nur die Bereitstellung von Hilfen ist notwendig, sondern vielmehr das aktive Eingreifen mithilfe von gesundheitsfördernden Maßnahmen durch die Institution Hochschule, in die die Studierenden frühzeitig integriert werden sollten.

Schlüsselwörter

Psychische Gesundheit - Psychische Belastung · Studierende · Medizin · Prävention . Prüfungsängstlichkeit · Psychische Störung

\section{Mental health of students. A comparison between nursing administration and health care management students of the University of Applied Sciences Zwickau and medical students of the University of Technology Dresden}

\section{Abstract}

Background. Studying at a university is a phase in life in which young adults are confronted with specific requirements. Medical students are considered to be particularly psychologically burdened. However, investigations comparing medical students with students of subjects in the same branch of industry are rare.

Objectives. The aim of the study was to examine and compare the mental health of medical students as well as of nursing administration and health care management students.

Materials and methods. Nursing administration and health care management students of the University of Applied Sciences Zwickau (WHZ) and medical students of the University of Technology Dresden (TU Dresden) were included. Various aspects of mental health were surveyed using an online questionnaire, which included the measuring instruments TAI-G, BSI-18 and to determine the subjectively perceived burden of the study. In all, 258 students participated in the survey.

Results. No significant differences with regard to the total score of test anxiety and psychological symptoms could be found between the two student groups. However, medical students indicated that they perceived a significantly higher study burden compared to the nursing administration and health care management students.
Conclusions. The total scores were partly higher than scores presented in comparable studies. The students should receive preventive support in order to develop their own coping strategies. Not only the provision of help is necessary, but also active intervention with the help of health-promoting and preventive measures from the universities. Students should be integrated into these measures at an early stage.

Keywords

Mental health - Psychological distress . Students · Medicine - Prevention - Test anxiety . Mental disorder
Werten hinterlegt, wobei höhere Werte auf eine stärkere Prüfungsangst hinweisen [25].

BSI-18. Die persönliche Situation der Studierenden wurde mithilfe des „Brief Symptom Inventory 18“ (BSI-18) von Derogatis analysiert, der Kurzform des „Brief Symptom Inventory“ (BSI). Un- tersucht wird die aktuelle psychische Belastung auf symptomatischer Ebene in den letzten 7 Tagen $[12,14]$. Das BSI-18 beinhaltet drei 6-Item-Skalen: Somatisierung (Chronbachs $\alpha=0,68$ ), Depressivität (Chronbachs $\alpha=0,85$ ) und Ängstlichkeit (Chronbachs $\alpha=0,80 ;[13$, 14]). Für die Auswertung wird ein Summenwert gebildet, der als „Global Severity
Index" (GSI) bezeichnet wird und das Ausmaß der psychischen Belastung widerspiegelt (Chronbachs $\alpha=0,89$; [23]).

\section{Subjektiv wahrgenommene Belastung} durch das Studium. Die aktuell wahrgenommene Belastung durch das Studium von den Studierenden wurde mithilfe eines Items ermittelt („Wie stark füh- 
Tab. 2 Ergebnisse derErhebungsinstrumente zur psychischen Gesundheit der Gesundheits-und Pflegemanagement-sowie derMedizinstudierenden im Vergleich (Mittelwert, Standardabweichung [in Klammern], Mann-Whitney-U-Test, Effektstärke Cohens d)

\begin{tabular}{|c|c|c|c|c|c|}
\hline & \multicolumn{2}{|l|}{ Studienfach } & \multirow{3}{*}{$\boldsymbol{U}$} & \multirow{3}{*}{$p$} & \multirow{3}{*}{$d$} \\
\hline & $\begin{array}{l}\text { Gesundheits- und } \\
\text { Pflegemanagement }\end{array}$ & Medizin & & & \\
\hline & $n=103$ & $n=155$ & & & \\
\hline \multicolumn{6}{|l|}{ Prüfungsängstlichkeit (TAI-G-Kurzversion) } \\
\hline Skala „Aufgeregtheit" & $8,5(3,3)$ & $8,8(3,3)$ & $7.528,0$ & 0,437 & 0,10 \\
\hline Skala „Besorgtheit” & $13,7(3,8)$ & $13,3(3,8)$ & $8.404,0$ & 0,471 & 0,09 \\
\hline Skala „Mangel an Zuversicht" & $7,7(2,2)$ & $7,5(2,0)$ & $8.462,5$ & 0,408 & 0,10 \\
\hline Skala „Interferenz" & $6,2(1,8)$ & $5,5(2,1)$ & $9.764,5$ & 0,002 & 0,39 \\
\hline Gesamtscore & $36,0(9,2)$ & $35,1(8,6)$ & $8.452,5$ & 0,423 & 0,10 \\
\hline \multicolumn{6}{|l|}{ Psychische Belastung (BSI-18) } \\
\hline Skala "Somatisierung" & $2,7(2,9)$ & $2,6(2,9)$ & $8.039,5$ & 0,912 & 0,01 \\
\hline Skala „Depressivität“ & $4,6(4,9)$ & $4,4(4,5)$ & $8.073,5$ & 0,876 & 0,02 \\
\hline Skala „Ängstlichkeit" & $3,6(3,3)$ & $4,6(3,9)$ & $6.907,0$ & 0,065 & 0,23 \\
\hline Global Severity Index (GSI) & $10,9(9,7)$ & $11,6(9,4)$ & $7.502,0$ & 0,412 & 0,10 \\
\hline \multicolumn{6}{|c|}{ Subjektiv wahrgenommene Belastung durch das Studium } \\
\hline Überhaupt nicht belastet (1) bis stark belastet (5) & $2,6(0,9)$ & $3,8(0,9)$ & $3.082,5$ & $<0,001$ & 1,21 \\
\hline
\end{tabular}

len Sie sich aktuell durch das Studium belastet?"). Die Belastung war auf einer fünfstufigen Likert-Skala von „überhaupt nicht belastet“ bis „stark belastet“ einzuschätzen. Diese Frage ist von besonderer Bedeutung, da sie neben der Messung durch die diagnostischen Verfahren auch die subjektive Wahrnehmung der Studierenden berücksichtigt.

\section{Statistische Auswertung}

Die statistische Auswertung wurde mittels IBM SPSS Statistics 24 durchgeführt. Zur Ermittlung der soziodemografischen Daten sowie der aus den Messinstrumenten resultierenden Ergebnisse erfolgte zunächst eine deskriptive Auswertung. Die Ergebniswerte wurden durch die jeweiligen arithmetischen Mittel $(\bar{x})$ und den zugehörigen Standardabweichungen $(S D)$ beschrieben.

Um signifikante Unterschiede hinsichtlich der psychischen Gesundheit zwischen den Stichproben feststellen zu können, wurden statistische Testverfahren angewendet. Es wurde ein Signifikanzniveau von $\alpha=0,05$ gewählt. Es erfolgte eine Prüfung der Normalverteilung mittels Shapiro-Wilk-Test. Da keine Normalverteilungen vorlagen, wurden Mann-Whitney-U-Tests berechnet. Zur weiteren Charakterisierung der
Mittelwertsunterschiede zwischen den Gruppen wurde die Effektstärke Cohens $d$ nach Tomczak [27] bestimmt. Nach Cohen gelten Effektstärken zwischen $d=0,2-0,5$ als klein, zwischen 0,5 und 0,8 als moderat und $>0,8$ als groß [5].

Die notwendige Stichprobengröße wurde mit $\mathrm{G}^{\star}$ Power 3.1.3 kalkuliert [10]. Für den Vergleich von zwei Gruppen mittels Mann-Whitney-U-Tests (Studierende der WHZ vs. Studierende der TU, einseitige Testung) ist bei einem Signifikanzniveau von $\alpha=0,05$, einer Effektstärke von Cohens $d=0,5$ und einer Power von $95 \%(1-\beta=0,95)$ eine Stichprobe von $n=92$ Personen pro Gruppe (Gesamt $n=184$ ) notwendig.

\section{Ergebnisse}

\section{Stichprobe}

Von insgesamt 421 Studierenden, welche im Rahmen dieser Untersuchung die Grundgesamtheit darstellten, nahmen 258 Studierende vollständig an der Befragung teil, was einem Anteil von 61,3\% entsprach. Von diesen waren 103 (39,9\%) Gesundheits- und Pflegemanagementstudierende der WHZ und 155 (60,1\%) Medizinstudierende der TU Dresden. Es nahmen bei den WHZ-
Studierenden 91 Frauen (88,3\%) und bei den TU-Studierenden 113 Frauen $(72,9 \%)$ an der Befragung teil. Das Durchschnittsalter der Teilnehmer lag in Zwickau bei 21,6 Jahren $(S D=4,3)$ und in Dresden bei 22,1 Jahren $(S D=3,7$; - Tab. 1).

\section{Prüfungsängstlichkeit (TAI-G- Kurzversion)}

Die Teilnehmer aus Zwickau erreichten hinsichtlich der Prüfungsängstlichkeit einen durchschnittlichen Gesamtscore von 36,0. Die untersuchten Studierenden der WHZ wiesen dabei deskriptiv geringfügig höhere Werte auf als die Studierenden an der TU Dresden, welche einen durchschnittlichen Gesamtscore von 35,1 erreichten ( $p=0,423$; - Tab. 2). Eine Ausnahme bildete hierbei die Subskala „Aufgeregtheit", bei welcher der Durchschnittswert von den Medizinstudierenden etwas höher war $(p=0,437)$. Einen signifikanten Unterschied gab es lediglich auf der Subskala „Interferenz", bei welcher die Zwickauer Studierenden von einem deutlich stärkeren Durchschnittswert betroffen waren $(p=0,002$; - Tab. 2). 


\section{Originalarbeit}

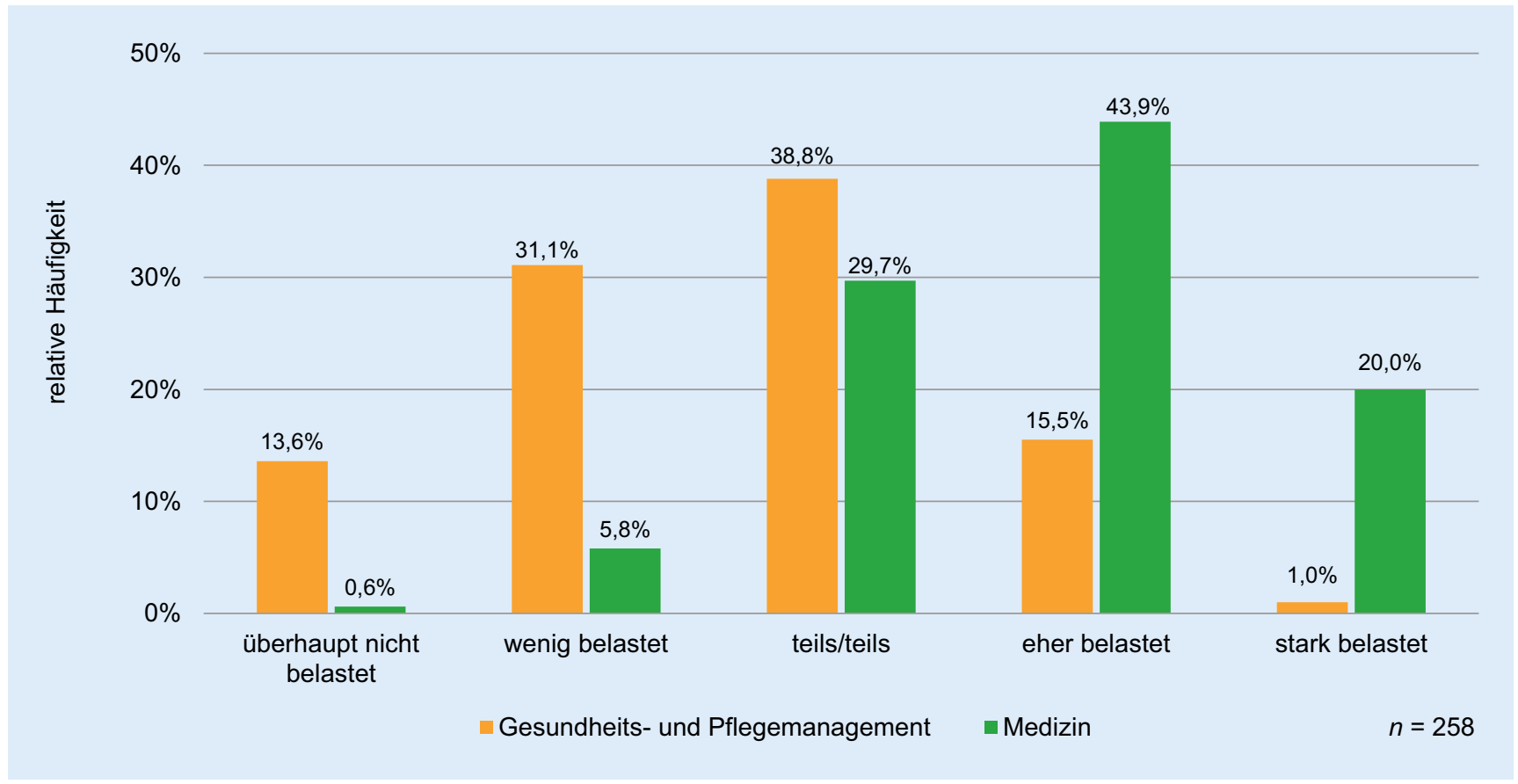

Abb. 1 ॥ Subjektiv wahrgenommene Belastung durch das Studium, Medizin- sowie Gesundheits- und Pflegemanagementstudierende im Vergleich

\section{Psychische Belastung (BSI-18)}

Im Hinblick auf die subjektiv empfundene Beeinträchtigung durch körperliche und psychische Symptome, welche durch das BSI-18 abgefragt wurden, ließ sich feststellen, dass die grundsätzliche psychische Belastung (GSI) mit 11,6 bei den Medizinstudierenden deskriptiv etwas höher war als bei den Gesundheitsund Pflegemanagementstudierenden mit 10,9 ( $p=0,412$; 0 Tab. 2).

Auch auf den Subskalen unterschieden sich die Studierenden hinsichtlich Somatisierung, Depressivität und Ängstlichkeit nicht-signifikant. Bei differenzierter Betrachtung der weiblichen Teilnehmer konnte hingegen ein signifikanter Unterschied zwischen beiden Studienfächern auf der Skala „Ängstlichkeit" festgestellt werden. Bei den weiblichen Studierenden in Dresden war ein deutlich höherer Durchschnittswert zu verzeichnen $(\bar{x}=4,9 ; S D=4,1)$ als bei den weiblichen Studierenden in Zwickau ( $\bar{x}=3,7$; $S D=3,4 ; p=0,041)$.

\section{Subjektiv wahrgenommene Belastung durch das Studium}

Im Gegensatz zu der Prüfungsängstlichkeit und der persönlichen Situation konnte bezüglich der subjektiv wahrgenommenen Studienbelastung ein hochsignifikanter Unterschied zwischen den Medizin- sowie den Gesundheits- und Pflegemanagementstudierenden ermittelt werden. Die Medizinstudierenden fühlten sich im Durchschnitt deutlich stärker durch ihr Studium belastet als die Zwickauer Studierenden $(p<0,001$; - Tab. 2). Der Effekt wies eine groß Effektstärke auf $(d=1,21)$. Zur besseren Veranschaulichung des Effekts gaben beispielsweise 20,0\% der Dresdner Studierenden an, sie fühlten sich stark durch das Studium belastet, während dies lediglich 1,0\% der Zwickauer Studierenden angaben (• Abb. 1).

\section{Diskussion}

Ziel der Untersuchung war es, die psychische Gesundheit der beiden Studierendengruppen der Studienfächer Gesundheits- und Pflegemanagement sowie Humanmedizin zu analysieren und vergleichend zu interpretieren.
Mithilfe der Untersuchung konnten keine signifikanten Unterschiede zwischen den Studierenden beider Studienfächer auf symptomatischer Ebene festgestellt werden. Die Erkenntnisse von Hope und Henderson [17] und Dyrbye et al. [7], die ein häufigeres Auftreten von Depressionen, Angst und Distress bei Medizinstudierenden beschreiben, konnten somit nicht bestätigt werden. Dieser Befund deckt sich mit aktuellen Studienergebnissen aus Deutschland [8]: In einem Vergleich von mehr als 4000 Studierenden unterschiedlicher Studienfächer von Medizin über Informatik bis Mathematik an vier Hochschulen gab es keine Unterschiede in der Ängstlichkeit oder Depressivität zwischen den Standorten.

Dennoch lieferten die Ergebnisse Hinweise dafür, dass weibliche Studierende psychisch stärker belastet sind als männliche Studierende, was im Hinblick aufHilfs- und Interventionsmaßnahmen nicht unberücksichtigt bleiben sollte. Die TAI-G-Gesamtscores der Studierenden lagen etwas über dem Vergleichswert von 34,6, den Wacker et al. [28] präsentieren. Beim Betrachten der persönlichen Situation, welche mittels BSI-18 gemessen wurden, fiel auf, dass die GSI- 
Werte deutlich über dem Vergleichswert von 6,3 liegen [23]. Die hohen GSIWerte geben Anlass für weitere Untersuchungen und das Hinzuziehen von professioneller Hilfe. Nichtsdestotrotz erreichten die Studierenden keine problematischen Werte $>20$, welche eine klinische Relevanz kennzeichnen [13].

Auch wenn auf symptomatischer Ebene mithilfe der Erhebungsinstrumente in dieser Untersuchung keine bedeutenden Unterschiede zwischen den Studienfächern festgestellt werden konnten, zeigte sie dennoch, dass sich die Dresdner Medizinstudierenden wesentlich stärker subjektiv durch ihr Studium belastet fühlten. Dies bestätigt die Erkenntnis von Bjorksten et al. [4], dass Medizinstudierende zwar den gleichen Anforderungen ausgesetzt sind, wie Studierende anderer Studienfächer, diese jedoch intensiver wahrnehmen und sich daher stärker belastet fühlen. Limitierend ist zu beachten, dass die Untersuchung von Bjorksten [4] bereits in den 1980er-Jahren durchgeführt wurde und dass in der hier präsentierten Studie das subjektive Belastungserleben nur mit einem Item operationalisiert werden konnte. Diese Erkenntnis gibt dennoch Anlass für weitere Untersuchungen, insbesondere nach den Ursachen, um Intervention gezielt einleiten zu können.

\section{Limitationen}

Auch wenn in dieser Studie eine im Vergleich zu anderen Erhebungsmethoden überdurchschnittlich hohe Rücklaufquote von $61,3 \% \mathrm{zu}$ verzeichnen war, stellen die insgesamt geringen Fallzahlen ein Defizit dar. Diese resultieren aus der geringen Grundgesamtheit von 421 Studierenden und der noch kleineren Teilnehmerzahl von 258 Studierenden, in denen die männlichen Studierenden zudem unterrepräsentiert sind. Des Weiteren wurde für jede Studierendengruppe jeweils nur ein Standort betrachtet. Durch dieses selektive Vorgehen wird die Repräsentativität und somit die Übertragbarkeit der Ergebnisse beeinträchtigt.

Eine weitere Limitation betrifft die Verwendung von retrospektiven Fragen, welche die Wahrscheinlichkeit eines Erinnerungsbias erhöhen [21]. Eine Schwä- che ist auch, dass sich einige Ergebnisse auf kurze Zeiträume beziehen, sodass starke Effekte unentdeckt bleiben können.

Bei den Fragebogeninhalten handelt es sich um komplexe Phänomene, die nicht durch die alleinige Anwendung der Instrumente abgebildet werden können. Um endgültige Schlüsse zu ziehen, müssen weitere Faktoren herangezogen werden. Ferner wurden verschiedene Instrumente miteinander kombiniert, um das mehrdimensionale Konstrukt „Psychische Gesundheit“ zu messen. Aufgrund der fehlenden einheitlichen Definition ist es schwierig, dieses Konstrukt $\mathrm{zu}$ operationalisieren und $\mathrm{zu}$ vergleichen. Dennoch wurden im Rahmen dieser Querschnittsanalyse mehrere Instrumente verwendet, mithilfe derer eine Einordnung der Ergebnisse möglich ist. Eine Ausnahme bildet die Variable „subjektiv wahrgenommene Belastung durch das Studium“, welche lediglich mit einem Item gemessen wurde.

\section{Fazit für die Praxis}

- Die Medizinstudierenden galten gemäß den Messinstrumenten psychisch zwar nicht als beeinträchtigter als die Gesundheits- und Pflegemanagementstudierenden, jedoch fühlten sie sich deutlich stärker durch ihr Studium belastet. Die Studierenden sollten deshalb bereits präventiv unterstützt werden, z. B. durch Unterstützungsangebote zum Thema Stressbewältigung oder Lernstrategien.

- Auch wenn die in der Studie ermittelten Gesamtscores nicht auf schwerwiegende psychische Beeinträchtigungen hindeuten, lagen sie teilweise deutlich über den Scores, die in vergleichbaren Studien präsentiert wurden. Deshalb ist nicht nur das Vorhalten von Hilfen notwendig, sondern vielmehr das aktive Eingreifen mithilfe von gesundheitsfördernden Maßnahmen durch die Institution Hochschule. Die Studierenden sollten zudem bereits frühzeitig in diese Maßnahmen integriert werden. Die Etablierung von gesundheitsfördern- den Settings muss also künftig mehr an Bedeutung gewinnen.

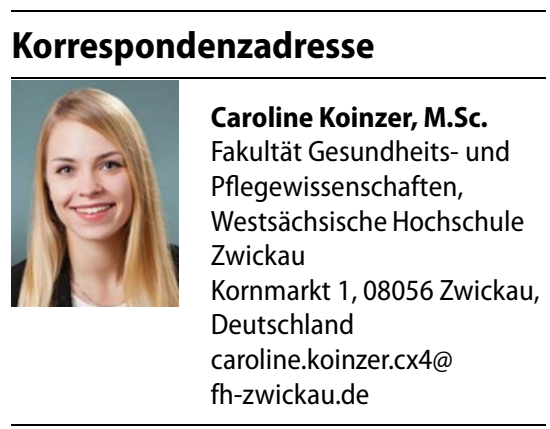

Funding. Open Access funding enabled and organized by Projekt DEAL.

\section{Einhaltung ethischer Richtlinien}

Interessenkonflikt. C. Koinzer, K. Hirsch, E.P. Richter und $\mathrm{H}$. Berth geben an, dass kein Interessenkonflikt besteht.

Für diesen Beitrag wurden von den Autoren keine Studien an Menschen oder Tieren durchgeführt. Für die aufgeführten Studien gelten die jeweils dort angegebenen ethischen Richtlinien.

Open Access. Dieser Artikel wird unter der Creative Commons Namensnennung 4.0 International Lizenz veröffentlicht, welche die Nutzung, Vervielfältigung, Bearbeitung, Verbreitung und Wiedergabe in jeglichem Medium und Format erlaubt, sofern Sie den/die ursprünglichen Autor(en) und die Quelle ordnungsgemäß nennen, einen Link zur Creative Commons Lizenz beifügen und angeben, ob Änderungen vorgenommen wurden.

Die in diesem Artikel enthaltenen Bilder und sonstiges Drittmaterial unterliegen ebenfalls der genannten Creative Commons Lizenz, sofern sich aus der Abbildungslegende nichts anderes ergibt. Sofern das betreffende Material nicht unter der genannten Creative Commons Lizenz steht und die betreffende Handlung nicht nach gesetzlichen Vorschriften erlaubt ist, ist für die oben aufgeführten Weiterverwendungen des Materials die Einwilligung des jeweiligen Rechteinhabers einzuholen.

Weitere Details zur Lizenz entnehmen Sie bitte der Lizenzinformation auf http://creativecommons.org/ licenses/by/4.0/deed.de.

\section{Literatur}

1. Bailer J, Schwarz D, Witthöft M, Stübinger C, Rist F (2008) Prävalenz psychischer Syndrome bei Studierenden einer deutschen Universität. Psychother Psychosom Med Psychol 58:423-429

2. Beiter R, Nash R, McCrady M, Rhoades D, Linscomb M, Clarahan M, Sammut S (2015) The prevalence and correlates of depression, anxiety, and stress in a sample of college students. J Affect Disord 173:90-96 


\section{Originalarbeit}

3. Bíró E, Adány R, Kósa K (2011) Mental health and behaviour of students of public health and their correlation with social support: a cross-sectional study. BMC Public Health 11:871

4. Bjorksten O, Sutherland S, Miller C, Stewart T(1983) Identification of medical student problems and comparison with those of other students. J Med Educ 58:759-767

5. Cohen J (1988) Statistical power analysis for the behavioural science. Erlbaum, Hillsdale

6. Dinkel A, Berth H, Balck F (2008) Belastungen und psychische Beschwerden von Medizinstudierenden: ein Überblick. In: Brähler E, Alfermann D, Stiller J (Hrsg) Karriereentwicklung und berufliche Belastung im Arztberuf. Vandenhoeck \& Ruprecht, Göttingen, S21

7. Dyrbye LN, Thomas MR, Shanafelt TD (2006) Systematic review of depression, anxiety, and other indicators of psychological distress among U.S. and Canadian medical students. Acad Med 81:354-373

8. Eissler C, Sailer M, Walter S, Jerg-Bretzke L (2020) Psychische Gesundheit und Belastung bei Studierenden. Präv Gesundheitsf 15:242-249

9. El Ansari W, Stock C, Mills C (2013) Is alcohol consumption associated with poor academic achievement in university students? Int J Prev Med 4:1175-1188

10. Erdfelder E, Faul F, Buchner A (1996) GPOWER: a general power analysis program. Behav Res Methods Instrum Comput 28:1-11

11. Europäische Kommission (EK) (2005) Grünbuch. Die psychische Gesundheit der Bevölkerung verbessern - Entwicklung einer Strategie für die Förderung der psychischen Gesundheit in der Europäischen Union. EK, Brüssel, S4

12. Franke GH (2000) BSl; BriefSymptom Inventory von L.R. Derogatis; Deutsche Version; Manual. Beltz, Göttingen, S14

13. Franke $G H$, Ankerhold $A$, Haase $M$, Jäger $S$, Tögel C, Ulrich C, Frommer J (2011) Der Einsatz des Brief Symptom Inventory 18 (BSI-18) bei Psychotherapiepatienten. Psychother Psychosom Med Psychol 61:82-86

14. Franke GH (2017) Mini-SCL. Mini-symptomchecklist. Hogrefe, Göttingen

15. Hodapp V (1991) Das Prüfungsängstlichkeitsinventar TAI-G: Eine erweiterte und modifizierte Version mit vier Komponenten. Z Pädagog Psychol 5:121-130

16. Hofmann F-H, Sperth M, Holm-Hadulla RM (2017) Psychische Belastungen und Probleme Studierender. Psychotherapeut 62:395-402

17. Hope V, Henderson M (2014) Medical student depression, anxiety and distress outside North America: a systematic review. Med Educ 48:963-979

18. Jacobi F, Becker M, Müllender S, Bretschneider J (2017) Epidemiologie psychischer Störungen. In: Möller H-J, Laux G, Kapfhammer H-P (Hrsg) Psychiatrie, Psychosomatik, Psychotherapie. Springer, Berlin, S138

19. Kirsch A-S, Laemmert P, Tittelbach S (2017) Gesundheitliche Anforderungen und Ressourcen von Studierenden. Eine Studie mit Studierenden der Sportökonomie. Präv Gesundheitsf 12:181-188

20. Lutz-Kopp C, Meinhardt-Injac B, Luka-Krausgrill U (2019) Psychische Belastung Studierender. Präv Gesundheitsf 14:256-263

21. Micheel H-G (2010) Quantitative empirische Sozialforschung. Reinhardt, München, S87

22. Spielberger CD (1980) Test Anxiety Inventory: Preliminary professional manual. Consulting PsychologistPress, Palo Alto
23. Spitzer C, Hammer S, Löwe B, Grabe HJ, Barnow S et al (2011) Die Kurzform des Brief Symptom Inventory (BSI-18); Erste Befunde zu den psychometrischen Kennwerten der deutschen Version. Fortschr Neurol Psychiat 79:517-523

24. Stock C (2017) Wie bedeutsam ist Gesundheit für den Studienerfolg von Studierenden? Präv Gesundheitsf 12:230-233

25. Szafranski DD, Barrera TL, Norton PJ (2012) Test anxiety inventory; 30 years later. Anxiety Stress Coping 25:667-677

26. Techniker Krankenkasse (2015) Gesundheitsreport 2015; Gesundheitvon Studierenden. TK, Hamburg, $\mathrm{S} 16$

27. Tomczak M, Tomczak E (2014) The need to report effect size estimates revisited. An overview of some recommended measures of effect size. Trends Sport Sci 1:19-25

28. Wacker A, Jaunzeme J, Jaksztat S (2008) Eine Kurzform des Prüfungsängstlichkeitsinventars TAI-G.ZPädagog Psychol 22:73-81 